\title{
Filling of charged cylindrical capillaries
}

\author{
Siddhartha Das,,${ }^{1}{ }^{*}$ Sourayon Chanda, ${ }^{2}$ J. C. T. Eijkel, ${ }^{3}$ N. R. Tas, ${ }^{4}$ Suman Chakraborty, ${ }^{5}$ and Sushanta K. Mitra ${ }^{6}$ \\ ${ }^{1}$ Department of Mechanical Engineering, University of Maryland, College Park, Maryland 20742, USA \\ ${ }^{2}$ Department of Mechanical Engineering, University of Alberta, Edmonton, Alberta, Canada T6G $2 G 8$ \\ ${ }^{3}$ BIOS, The Lab-on-a-Chip Group, MESA+Institute for Nanotechnology, University of Twente, P.O. Box 217, \\ 7500 AE Enschede, The Netherlands \\ ${ }^{4}$ Transducers Science and Technology, MESA+ Institute for Nanotechnology, University of Twente, P.O. Box 217, \\ 7500 AE Enschede, The Netherlands \\ ${ }^{5}$ Department of Mechanical Engineering, Indian Institute of Technology, Kharagpur-721302, India \\ ${ }^{6}$ Department of Mechanical Engineering, Lassonde School of Engineering, York University, Toronto, Ontario, Canada M3J1P3
}

(Received 27 October 2013; revised manuscript received 5 September 2014; published 16 October 2014)

\begin{abstract}
We provide an analytical model to describe the filling dynamics of horizontal cylindrical capillaries having charged walls. The presence of surface charge leads to two distinct effects: It leads to a retarding electrical force on the liquid column and also causes a reduced viscous drag force because of decreased velocity gradients at the wall. Both these effects essentially stem from the spontaneous formation of an electric double layer (EDL) and the resulting streaming potential caused by the net capillary-flow-driven advection of ionic species within the EDL. Our results demonstrate that filling of charged capillaries also exhibits the well-known linear and Washburn regimes witnessed for uncharged capillaries, although the filling rate is always lower than that of the uncharged capillary. We attribute this to a competitive success of the lowering of the driving forces (because of electroviscous effects), in comparison to the effect of weaker drag forces. We further reveal that the time at which the transition between the linear and the Washburn regime occurs may become significantly altered with the introduction of surface charges, thereby altering the resultant capillary dynamics in a rather intricate manner.
\end{abstract}

DOI: 10.1103/PhysRevE.90.043011

PACS number(s): 47.55.nb, 47.57.jd

\section{INTRODUCTION}

For close to a century, starting from the pioneering works of Lucas [1], Washburn [2], and Bosanquet [3], the filling dynamics of thin capillaries has attracted immense attention of the fluid mechanics community. The attention has stemmed from various reasons: First, the capillary filling process is an ideal example of low Reynolds number flow; second, such filling and the resulting capillary dynamics is extremely crucial to understand biofluid transport in living organisms [4-9]; and third, this filling process is intrinsic to several industrial and scientific applications [10-14]. The central understanding of capillary filling problems is that the filling occurs by a balance of the driving surface tension and the retarding viscous forces. This leads to the celebrated Washburn regime, where $\ell \sim \sqrt{t}$ ( $\ell$ and $t$ are the filling length and the filling time, respectively) $[2,3,15-22]$. Recently, there have been efforts to look beyond the classical Washburn regime. For example, researchers have identified a linear or an inertial regime (where $\ell \sim t$ ) preceding the Washburn regime [23-31], and an oscillatory regime (witnessed in capillary rise problems) replacing the Washburn regime [15,23,32-37]. In fact, there can even be a prelinear regime, in case one accounts for the capillary end effects $[38,39]$. In addition, capillary filling has been studied in a variety of other systems, such as in porous media [40-44], in multiple fluid systems [45-48], in granular media [49], in irregular or noncircular geometries [50,51], etc.

Despite such overwhelming attention on different issues related to capillary filling, problems on filling of charged capillaries have remained relatively unexplored. This is especially

*sidd@umd.edu surprising given the large number of applications that require prefilling of charged capillaries. For example, the entire science of electro-osmosis in microfluidic transport is based on prefilling of charged capillaries with an electrolyte solution. In fact, the filling of charged capillaries represents a small subset of the massively explored domain of electrocapillary problems [52-58]. A direct experimental study that pinpoints the role of surface charge on capillary filling is still missing. Only a handful of experimental studies provided an indirect reference to the possible implications of wall charge on capillary filling $[59,60]$. These studies $[59,60]$ reported filling of glass nanochannels by de-ionized water and observed a filling rate smaller than that in an uncharged capillary. This reduction was hypothesized to occur due to a possible retarding electrical effect, caused by the spontaneous deprotonation of the capillary wall during the filling process. Unfortunately, a comprehensive mathematical analysis of the problem is absent. The only major related theoretical study is by Phan et al. [61], who calculated the streaming potential and the electroviscous effects [62-68] during the filling of charged nanochannels. This study, however, suffered from three key limitations. First, it did not consider the effect of the streaming-potential-induced retarding electro-osmotic transport in modifying the drag force on the filling liquid column. Second, it did not account for the overlap of the electric double layer or EDL. Such an overlap, which occurs when the EDL thickness $\lambda$ is larger than the capillary radius or nanochannel half height $[69,70]$, may become extremely important in nanoscale capillary filling. Third, it did not pinpoint the role of surface charge in affecting the different regimes (linear or Washburn) during the filling process. In this context, the studies of Jain and Chakraborty [71] and Waghmare and Mitra [20] are worth mentioning. In these studies, the authors investigated the combined effect of 
$p \mathrm{H}$ (neutral or acidic) and applied transverse electric field in dictating the capillary filling dynamics. However, in these studies, too, the distinctive aspects of the filling dynamics of charged capillaries relative to the uncharged ones remained unaddressed.

In the present study, we provide a closed-form analytical solution for describing the filling dynamics of charged horizontal capillaries. The inner walls of the capillary can be either originally charged (i.e., charged in air) or may acquire charge in contact with the filling liquid. The filling liquid is considered to be an electrolyte solution containing dissociated salt ions. Therefore, in response to charge on the wall, for both these cases, an EDL develops. The mobile ions within this EDL are transported downstream by the capillary flow. This eventually leads to the generation of an electric field, known as the streaming potential. This electric field has two closely related effects. First, it leads to a retarding electrical force (opposing the capillary drive) on the filling liquid column. Second, it modifies the flow profile by triggering an electro-osmotic transport opposing the capillary flow. This leads to a modification of the viscous wall drag force, because of an alteration of the velocity gradient at the capillary wall. We can connect such dual consequences of the electric field with the classical notion of the streaming-potentialinduced electroviscous effects [62-65,72,73]. In the presence of the streaming potential, an induced electro-osmotic flow, opposing the driving pressure-driven transport, sets in. In the present study, the consequent modified velocity field is used to compute the net wall drag force on the liquid column, with the liquid column being considered as a lumped mass. Such a lumped-mass-based description of the filling column column would imply that we must additionally account for the electrical force. This force is simply the unbalanced EDL charge (in the liquid column) times the electric field. This dual effect of the electric field is analogous to the dual effect of the surface tension force. The surface tension, because of the curvature of the air-liquid meniscus of the filling liquid column, triggers a pressure gradient that drives a flow field. This flow field exerts a drag force on the liquid column. At the same time, the surface tension force induces a pulling force on the filling liquid column that is treated as a lumped mass.

We study the charged capillary filling dynamics based on certain simplifying assumptions. First, we consider that the wall charge and the induced EDL electrostatics do not affect the dynamic contact angle that governs the capillary drive- the role of such a charge-affected dynamic contact angle has been discussed elsewhere [53]. Second, we consider that the EDL and the resulting electrostatic potential and the ion number density distribution are strictly one dimensional (i.e., varying only in the radial direction), and neglect the EDL in the wedge formed at the three-phase contact line [74-79]. Third, we neglect the charge accumulation at the air-liquid meniscus of the filling column. We discuss the implications and validity of this particular assumption in great detail in Sec. III D. Fourth, we restrict our analysis to capillaries of radii much larger than $10 \mathrm{~nm}$, so that we may neglect noncontinuum effects such as the inhomogeneous viscosity (near the walls), layering of the liquid molecules, etc. Finally, we neglect effects such as finite ion size [80-84], solvent polarization [85,86], etc., that may become significant in a nanoscale capillary.
Results show that the filling rate for a charged capillary is substantially smaller as compared to an uncharged capillary. This is characterized by a smaller dimensionless filling length $\bar{\ell}$ for a given dimensionless filling time $\bar{t}$, although the inertial and the Washburn regimes are present for the charged capillary as well. Such a reduced filling rate indicates that the effect of retarding electrical force on the filling liquid column outweighs the influence of reduced drag caused by the back electro-osmotic transport. Another important result is the role of capillary wall charges in dictating the transition from the inertial to the Washburn regime. We find that the time needed for this transition significantly increases for the charged capillary, as compared to the uncharged capillary. Also this increase is a monotonic function of the dimensionless ionic Peclet number and dimensionless wall potential [63], but a nonmonotonic function of the dimensionless EDL thickness. On the contrary, the equivalent filling length over which this transition occurs shows a distinctly nonintuitive behavior. For example, for a smaller dimensionless EDL thickness, this transition filling length is larger, whereas for larger extents of the EDL overlap, it is distinctly smaller than that corresponding to the uncharged capillary. This final result can be explained by unraveling the role of the additional electrical force in altering the slope of the $\ell$-vs- $t$ variation for the linear inertial regime and the alteration of the drag coefficient by considering the retarding electro-osmotic transport.

\section{THEORY}

We consider the filling of a charged circular capillary as shown in Fig. 1. The instantaneous filling length is denoted as $\ell$ and the corresponding filling time is considered as $t$. The $\ell$-vs- $t$ relationship can be obtained from the force balance on the liquid column (see Fig. 1):

$$
\frac{d}{d t}(m u)=F_{c}+F_{D}+F_{e} .
$$

In Eq. (1), $m=\pi R^{2} \rho \ell$ ( $R$ is the capillary radius and $\rho$ is the liquid density) is the mass of the liquid column, $u=\frac{d \ell}{d t}$ is the average velocity of the liquid column (which is also the filling rate), $F_{c}=2 K_{1} \pi R \cos \theta \gamma$ ( $\gamma$ is the surface tension, $\theta$ is the dynamic contact angle [37], and $K_{1}$ is the factor that accounts for the variation in the driving capillary forces due to effects such as precursor film formation, surface roughness, etc. [37])

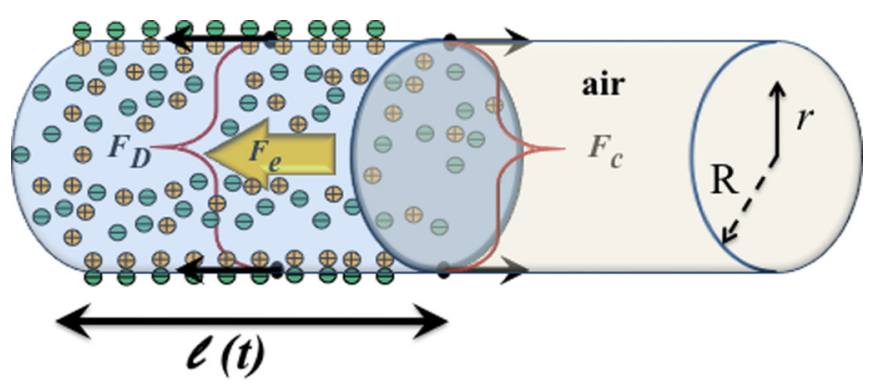

FIG. 1. (Color online) Schematic of an electrolytic solution filling a charged capillary. The schematic also shows the different forces on the filling liquid column. The circular boundary of the half-shaded region is the three-phase contact line. 
is the driving capillary force, $F_{D}=2 \pi R \ell \eta(\partial u / \partial r)_{r=R}=$ $-K_{2} \pi \eta \ell(d \ell / d t)$ ( $\eta$ is the dynamic viscosity and $K_{2}$ is the prefactor that depends on the functional form of the velocity profile; for example, for a fully developed Poisseuille flow, $K_{2}=8$ [37] $)$ is the retarding drag force, and $F_{e}=q_{p} E_{S}\left(q_{p}\right.$ is the net unbalanced charge contained in the liquid column and $E_{S}$ is the induced streaming electric field; both of these quantities are described in detail later) is the net electrical force. As a consequence, we can rewrite Eq. (1) as

$$
\pi R^{2} \rho \frac{d}{d t}\left(\ell \frac{d \ell}{d t}\right)=2 K_{1} \pi \cos \theta \gamma R-K_{2} \pi \eta \ell \frac{d \ell}{d t}+q_{p} E_{S} .
$$

Here we shall first describe $q_{p}$ and $E_{S}$. To do so, we need the EDL potential distribution $\psi$. Therefore, we start with the calculation of $\psi$.

Calculation of the EDL potential $\psi$. Both $q_{p}$ and $E_{S}$ depend on the EDL potential $\psi$ (see below). To obtain $\psi$, we invoke the one-dimensional Poisson-Boltzmann equation in cylindrical coordinates, so that we may write

$$
\frac{1}{r} \frac{d}{d r}\left(r \frac{d \psi}{d r}\right)=-\frac{e z\left(n_{+}-n_{-}\right)}{\epsilon_{0} \epsilon_{r}}=\frac{2 e z n_{\infty}}{\epsilon_{0} \epsilon_{r}} \sinh \left(\frac{e z \psi}{k_{B} T}\right) .
$$

In the previous equation, we assumed that the ionic number densities $n_{ \pm}$are governed by the Boltzmann distribution that can be expressed as

$$
n_{ \pm}=n_{\infty} \exp \left(\mp \frac{e z \psi}{k_{B} T}\right) .
$$

In the above equations, $n_{\infty}$ is the number density of both the cations and the anions in the bulk, $\psi$ is the EDL electrostatic potential, $z$ is the ion valence (we consider a symmetric electrolyte), $e$ is the electronic charge, $k_{B} T$ is the thermal energy, $\epsilon_{0}$ is the permittivity of free space, and $\epsilon_{r}$ is the relative permittivity of the medium. We would like to emphasize here that Eq. (4) governing the ion distribution remains valid even for the case of small enough capillary radius where the EDLs may overlap (i.e., $\lambda / R \geqslant 1$, where $\lambda=$ $\sqrt{\epsilon_{0} \epsilon_{r} k_{B} T / 2 n_{\infty} e^{2} z^{2}}$ is the EDL thickness). For such a case, the capillary centerline electrostatic potential is $\psi^{c}$ (where $\psi^{c} \neq 0$ ), and the corresponding ionic number densities are $n_{ \pm}=n_{ \pm}^{c} \exp \left[\mp e z\left(\psi^{c}-\psi\right) / k_{B} T\right]$ (where $n_{ \pm}^{c}$ are the capillary centerline ionic number densities). Assuming that the capillary is connected to a bulk electrolyte reservoir, we can relate $n_{ \pm}^{c}$ and $\psi^{c}$ to the corresponding (known) values at this reservoir (where $n_{\infty}$ and $\psi=0$ are the ionic number densities and the EDL potential) as $n_{ \pm}^{c}=n_{\infty} \exp \left(\mp e z \psi^{c} / k_{B} T\right)$, so that we do get $n_{ \pm}=n_{\infty} \exp \left(\mp e z \psi / k_{B} T\right)$. Therefore, Eq. (4) is equally valid for the case of overlapped EDLs, as long as $n_{\infty}$ is the ion number density in the reservoirs connecting the charged capillary.

We can obtain an analytical solution of Eq. (3) for the case where the capillary wall potential $\left(\psi_{0}\right)$ is small $\left(\left|\psi_{0}\right| \leqslant\right.$ $25 \mathrm{mV}$ ), so that we can apply Debye-Hückel linearization [73] to reduce Eq. (3) to

$$
\frac{1}{r} \frac{d}{d r}\left(r \frac{d \psi}{d r}\right)=\frac{\psi}{\lambda^{2}}
$$

We solve Eq. (5) in the presence of the condition $\psi(r=R)=$ $\psi_{0}$ and $d \psi / d r(r=0)=0$ to obtain $\psi$ in dimensionless form as

$$
\bar{\psi}=\bar{\psi}_{0} \frac{I_{0}(\bar{r} / \bar{\lambda})}{I_{0}(1 / \bar{\lambda})},
$$

where $\bar{\psi}=e z \psi / k_{B} T, \bar{\psi}_{0}=e z \psi_{0} / k_{B} T, \bar{r}=r / R, \bar{\lambda}=\lambda / R$, and $I_{0}(x)$ is the modified Bessel function of the first kind of order 0 and argument $x$. Please note we also perform a full-scale numerical solution of the EDL potential distribution [see Eq. (3)], valid for any general $\psi_{0}$. The numerical solution is performed by using the built-in functionODE45 in MATLAB. ODE45 employs the adaptive step size Runge-Kutta (fourth-/fifth-order) method for solving an ordinary differential equation numerically.

Calculation of $q_{p} . q_{p}$ is the net unbalanced charge contained in the liquid column. This charge is the charge contained in the EDL formed in response to the charge on the inner walls of the capillary. Such charge on the capillary walls can be either intrinsically present (i.e., the walls are charged in air) or may be acquired because of de-ionization when the filling liquid comes in contact with the capillary wall. In the case where the filling liquid is an electrolyte, the electrolyte ions will be attracted towards the wall charge, thereby forming the EDL. Such an EDL may form even when the liquid does not contain electrolyte ions. For such a case the ions in the EDL will be the ions obtained from the dissociation of the liquid (e.g., $\mathrm{H}_{3} \mathrm{O}^{+}$and $\mathrm{OH}^{-}$ions obtained from the dissociation of water). The assumption that an EDL forms instantaneously after the liquid column comes in contact with the charged solid is based on the large difference between the EDL formation time scale ( $\tau_{\mathrm{EDL}}$ ) and the capillary filling time scale. $\tau_{\mathrm{EDL}} \sim \lambda^{2} / D$ (where $\lambda \sim 1-100 \mathrm{~nm}$ and $D \sim 10^{-9} \mathrm{~m}^{2} / \mathrm{s}$ is the ion diffusivity), so that $\tau_{\mathrm{EDL}}=10^{-9}-10^{-5} \mathrm{~s}$-hence the EDL formation time scale is substantially smaller than the capillary filling time scale. A similar order of magnitude difference must also exist between the capillary filling time scale and the time scale of the chemical reactions at the capillary wall. These reactions become important when the capillary wall acquires charges owing to de-ionization in contact with the filling liquid. There is another assumption implicit in the derivation of Eq. (4): We assume that the ions that form the EDL in the capillary (either the electrolyte ions or the ions obtained from the dissociation of water or the de-ionization of the solid) must also be present in the reservoir connecting the capillary. Of course, this assumption necessarily disregards the fact that due to the chemical reactions at the capillary walls, these ions may be present in different forms within the capillary and the reservoir. For the case when there is an intrinsic charge on the capillary wall, the imbalance in the number densities between the coions and counterions within the EDL is accounted for by the fact that the capillary is connected to an infinite reservoir that takes care of this imbalance. In other words, this imbalance is not an issue, since the capillary is not an isolated system; rather, it is connected to an infinite reservoir of the electrolyte. For the case where the wall attains the charge on because of de-ionization in contact with the filling liquid, the wall charge (and hence the wall potential) is a function of the ion concentration and the $p \mathrm{H}$ of the filling liquid [72,87-90]. There are well-known relationships connecting the wall potential as a function of the 
ion concentration and buffer $p \mathrm{H}$. Of course, we do not use any of these relationships; rather, we consider only a parametric variation of $\psi_{0}$ and ion concentration (or EDL thickness). To make such a scenario perfectly feasible even for this case (where the wall potential depends on ion concentration and buffer $p \mathrm{H}$ ), one needs to operate at a specified buffer $p \mathrm{H}$, which ascertains our desired combination of wall potential and ion concentration or EDL thickness (for more details, kindly refer to Das et al. [72]).

The net unbalanced charge contained in the liquid column, which is the net unbalanced charge in the EDL, can be expressed as [using Eqs. (4) and (6)]

$$
\begin{aligned}
q_{p} & =\ell \int_{0}^{R} e z\left(n_{+}-n_{-}\right) 2 \pi r d r=-\ell \frac{4 \pi n_{\infty} e^{2} z^{2}}{k_{B} T} \int_{0}^{R} \psi r d r \\
& =-4 \pi \ell n_{\infty} e z R^{2} \int_{0}^{1} \bar{\psi} \bar{r} d \bar{r}=q_{0}\left[-\bar{\psi}_{0} \frac{1}{\bar{\lambda}} \frac{I_{1}(1 / \bar{\lambda})}{I_{0}(1 / \bar{\lambda})}\right], \quad(7)
\end{aligned}
$$

where $I_{1}(x)$ is the modified Bessel function of the first kind of order 1 and $q_{0}=2 \pi \epsilon_{0} \epsilon_{r} k_{B} T \ell / e z$ has the units of charge. $I_{1}(1 / \bar{\lambda}) \rightarrow 0$ and $I_{0}(1 / \bar{\lambda}) \rightarrow 0$ when $1 / \bar{\lambda} \rightarrow 0$, i.e., $\bar{\lambda} \rightarrow \infty$. Using the condition that $\left[I_{n}(x)\right]_{x \rightarrow 0} \approx \frac{1}{n !}\left(\frac{x}{2}\right)^{n}$, we can obtain the limiting value of $q_{p}$ from Eq. (7) [for the case when $1 / \bar{\lambda} \rightarrow$ 0 , i.e., the case when both $I_{1}(1 / \bar{\lambda}) \rightarrow 0$ and $I_{0}(1 / \bar{\lambda}) \rightarrow 0$ ] as

$$
\left(q_{p}\right)_{1 / \bar{\lambda} \rightarrow 0} \approx\left(-q_{0} \bar{\psi}_{0} \frac{1}{2 \bar{\lambda}^{2}}\right)_{1 / \bar{\lambda} \rightarrow 0}=0 .
$$

The fact that the net unbalanced charge goes to zero for large dimensionless EDL thickness values (i.e., when $1 / \bar{\lambda} \rightarrow 0$ ) is clearly evident from the figure in the inset of Fig. 2(a). Physically, such a behavior can be explained by noting that when $\bar{\lambda} \rightarrow \infty$, i.e., there is a large EDL overlap, we have $\lambda \rightarrow$ $\infty$ and/or $R \rightarrow 0$. The former case would mean a negligible ion number density (since $n_{\infty} \sim 1 / \lambda^{2}$ ), whereas the latter case would imply a negligible liquid volume. For either of the cases, therefore, it is intuitive that the net charge content in the liquid volume will be negligible. Such a dependence is also evident from Eq. (7), which clearly shows that $q_{p} \sim R^{2}$ and $q_{p} \sim n_{\infty}$. Please note that for generalized $\psi_{0}$, i.e., where we do not have an analytical solution of $\psi$, we obtain $q_{p}$ numerically from the relationship [see Eq. (7)] $q_{p}=-4 \pi \ell n_{\infty} e z R^{2} \int_{0}^{1} \bar{\psi} \bar{r} d \bar{r}$.

An important assumption intrinsic to Eq. (7) is about the one dimensionality of the EDL potential $\psi$ and the ionic concentration distribution, i.e., we assume that all these variables only vary with the radial coordinate $r$. This is significant in light of the fact that recent studies [61] have suggested that in filling of charged capillaries there must be an accumulation of unequal ion concentration at the moving interface. This would mean that $\psi$ and $n_{ \pm}$would also be a function of the axial coordinate $x$. However, we neglect the contribution of such a distribution in the calculation presented in Eq. (7). We have provided a detailed discussion later (see Sec. III D) on the significance and validity of the assumption of neglecting ion accumulation at the moving interface.

Calculation of $E_{S}$. To obtain $E_{S}$, we make the net ionic current equal to zero, i.e.,

$$
I=e z \int_{0}^{R}\left(n_{+} u_{+}-n_{-} u_{-}\right) 2 \pi r d r=0 .
$$
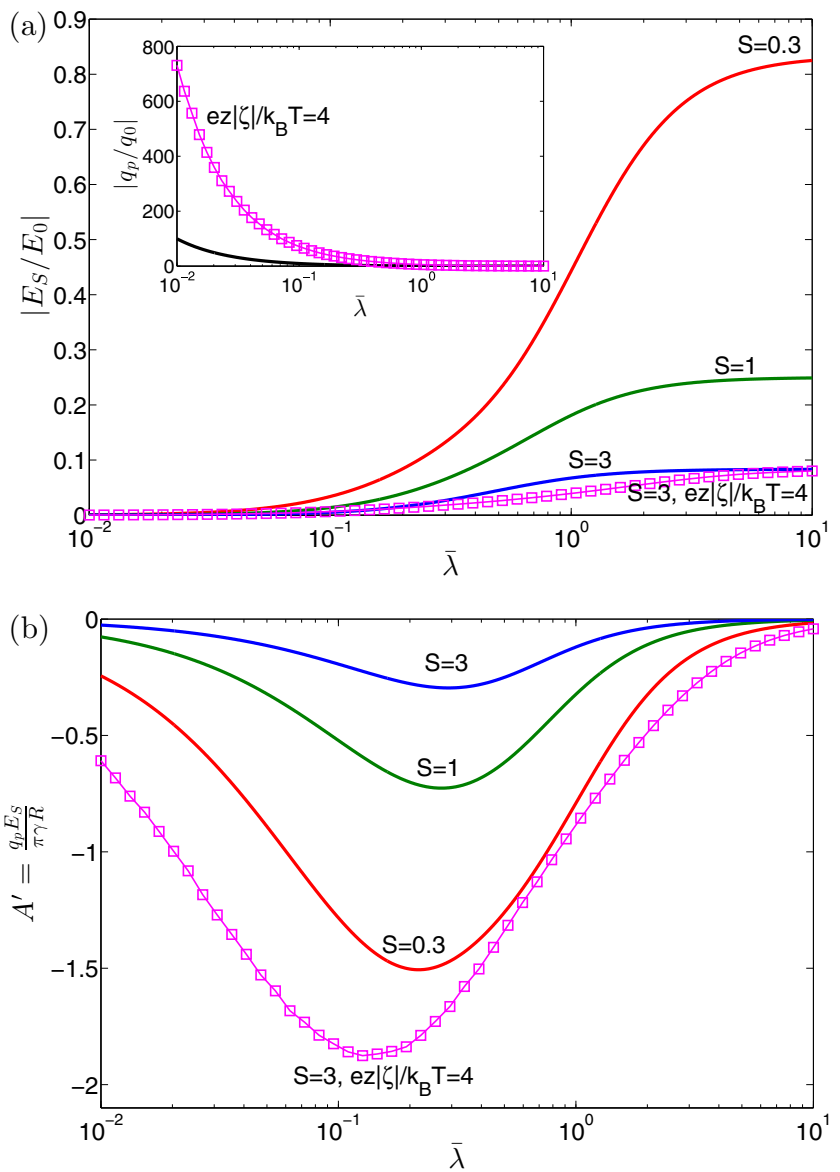

FIG. 2. (Color online) (a) Variation of the magnitude of the dimensionless streaming potential $\left|E_{S} / E_{0}\right|$ with dimensionless EDL thickness $\bar{\lambda}$ with different values of the ionic Peclet number $S$. In the inset of the figure, we show the variation of the magnitude of the dimensionless charge $\left|q_{p} / q_{0}\right|$ with $\bar{\lambda} .\left|q_{p} / q_{0}\right|$ is independent of $S$. (b) Variation of the dimensionless ratio $A^{\prime}=q_{p} E_{S} / \pi \gamma R$ with $\bar{\lambda}$ for different values of $S$. For both plots, except for the case where $\left|\bar{\psi}_{0}\right|=e z\left|\psi_{0}\right| / k_{B} T$ is specified, we always take $\left|\bar{\psi}_{0}\right|=1$. For the case where $\left|\bar{\psi}_{0}\right|=4$, we obtain the results numerically.

Here $u_{ \pm}$are the ionic velocities expressed as

$$
u_{ \pm}=u_{\mathrm{adv}}+u_{\mathrm{cond}, \pm}=\left(u_{P}+u_{E}\right) \pm e z E_{S} / f
$$

where $u_{\text {cond, } \pm}= \pm e z E_{S} / f$ are the ionic conduction velocities. Here $f$ is the ionic friction factor, assumed identical for the cations and anions [63-65]. Please note that the analysis is equally possible in case $f$ is not identical for the cations and anions. Also $u_{\mathrm{adv}}=u_{P}+u_{E}$ is the ion advection velocity. $u_{\text {adv }}$ is identical for cations and anions and is a linear combination of the pressure-driven velocity $\left(u_{P}\right)$ and streamingpotential-induced electro-osmotic velocity $\left(u_{E}\right)$. Considering the velocity field to be steady and fully developed, we can write

$$
\begin{aligned}
& u_{P}=-\frac{1}{4 \eta}(d p / d x) R^{2}\left(1-\bar{r}^{2}\right), \\
& u_{E}=-\frac{\epsilon_{0} \epsilon_{r} \psi_{0} E_{S}}{\eta}\left(1-\bar{\psi} / \bar{\psi}_{0}\right),
\end{aligned}
$$


where $-d p / d x$ is the applied pressure gradient. Using Eqs. (10) and (11) in Eq. (9), we finally get [using $-d p / d x=$ $(2 \gamma / R) / \ell$ and Eqs. (4) and (6)]

$$
\begin{aligned}
E_{S} & =\frac{\frac{R(z e)^{2}}{\epsilon_{0} \epsilon_{r}\left(k_{B} T\right)^{2}}(\gamma / \ell) \int_{0}^{R} r\left(1-r^{2} / R^{2}\right) \psi d r}{\frac{\eta(R z e)^{2}}{f \epsilon_{0} \epsilon_{r} k_{B} T}+\frac{(e z)^{2} \psi_{0}}{\left(k_{B} T\right)^{2}} \int_{0}^{R} 2 \psi\left(1-\psi / \psi_{0}\right) r d r} \\
& =E_{0}\left[\frac{\bar{\psi}_{0} \bar{\lambda}^{2} I_{2}(1 / \bar{\lambda}) / I_{0}(1 / \bar{\lambda})}{S / 2+\bar{\psi}_{0}^{2} M_{B}}\right],
\end{aligned}
$$

where

$$
M_{B}=\frac{I_{0}^{2}(1 / \bar{\lambda})}{I_{1}^{2}(1 / \bar{\lambda})}-\frac{I_{2}(1 / \bar{\lambda})}{I_{0}(1 / \bar{\lambda})}
$$

Here $E_{0}=\frac{2 e z \gamma R}{\epsilon_{0} \epsilon_{r} k_{B} T \ell}$ has the units of electric field and $S=$ $\frac{2 e^{2} z^{2} \eta}{\epsilon_{0} \epsilon_{r} k_{B} T f}$ is the dimensionless ionic Peclet number [63]. Similar to $q_{p}$, for generalized $\psi_{0}, E_{S}$ is obtained numerically from the relation

$$
E_{S}=\frac{\frac{R(z e)^{2}}{\epsilon_{0} \epsilon_{r}\left(k_{B} T\right)^{2}}(\gamma / \ell) \int_{0}^{R} r\left(1-r^{2} / R^{2}\right) \psi d r}{\frac{\eta(R z e)^{2}}{f \epsilon_{0} \epsilon_{r} k_{B} T}+\frac{(e z)^{2} \psi_{0}}{\left(k_{B} T\right)^{2}} \int_{0}^{R} 2 \psi\left(1-\psi / \psi_{0}\right) r d r} .
$$

Calculation of the electrical force $q_{p} E_{S}$. The electrical force $F_{e}=q_{p} E_{S}$ on the liquid column [see Eq. (2)] can be expressed as [using Eqs. (7) and (12)]

$$
F_{e}=q_{p} E_{S}=-4 \pi \gamma R \frac{\bar{\psi}_{0}^{2} \bar{\lambda}}{S / 2+\bar{\psi}_{0}^{2} M_{B}} \frac{I_{2}(1 / \bar{\lambda}) I_{1}(1 / \bar{\lambda})}{\left[I_{0}(1 / \bar{\lambda})\right]^{2}} .
$$

For generalized $\psi_{0}, F_{e}$ can be obtained from the numerical values of $q_{p}$ and $E_{S}$. It is worthwhile to discuss here the origin of this retarding electrical force $F_{e}=q_{p} E_{S}$ on the filling liquid column. The streaming potential is fundamentally created whenever there is an axial charge separation by the advection of any fluid mass with a net charge imbalance. Such an advection first generates the well-known streaming current. However, since there is no externally employed electric field, the net current within the system has to be zero. This ultimately results in the generation of an electric field $E_{S}$, with $E_{S}$ being directed opposite to the direction of the advective transport. For the present case, during the filling process there is a continuous advection of a mass of liquid with a net charge imbalance (the EDL is the source of this charge imbalance). Hence, by applying the principle of generation of the streaming potential, we can safely assume that there will always be a streaming potential accompanied with the process of filling of charged capillaries. It is well known that in the presence of the streaming potential, the per unit volume body force term used in the Navier-Stokes equation describing the fluid flow is $\rho_{e} E_{s}=e z\left(n_{+}-n_{-}\right) E_{S}$. Therefore, when we consider the force on the total mass of the filling liquid column, we need to integrate this force over the entire volume of the filling liquid column, and that force is thus $\ell \int_{0}^{R} e z\left(n_{+}-n_{-}\right) 2 \pi r E_{S} d r=$ $\left[\ell \int_{0}^{R} e z\left(n_{+}-n_{-}\right) 2 \pi r d r\right] E_{S}=q_{p} E_{S}=F_{e}$ [see Eq. (7)].

Calculation of the drag force. The drag force on the liquid column can be expressed as

$$
F_{D}=2 \pi R \ell \eta(\partial u / \partial r)_{r=R} .
$$

Therefore, to obtain the drag force, we need the velocity profile. In classical capillary filling studies [2,3,15-22,59], the drag force has been computed considering a fully developed pressure-driven Poisseuille flow profile:

$$
u=u_{P}=-\frac{1}{4 \eta} \frac{d p}{d x} R^{2}\left(1-\bar{r}^{2}\right)=2 u_{\mathrm{avg}}\left(1-\bar{r}^{2}\right)
$$

where

$$
u_{\mathrm{avg}}=\frac{\int_{0}^{R} u_{P} 2 \pi r d r}{\int_{0}^{R} 2 \pi r d r}=-\frac{1}{8 \eta} \frac{d p}{d x} R^{2} .
$$

Therefore, using Eq. (16) in Eq. (15), we shall get (using $\left.u_{\text {avg }}=d \ell / d t\right)$

$$
F_{D}=-8 \pi \eta \ell \frac{d \ell}{d t}=-K_{2} \pi \eta \ell \frac{d \ell}{d t},
$$

which implies $K_{2}=8$ [see Eq. (2)].

For the present case, this simple formulation needs to be modified, since now the flow is a fully developed combined pressure-driven and electro-osmotic transport:

$$
u=u_{P}+u_{E}=-\frac{1}{4 \eta} \frac{d p}{d x} R^{2}\left(1-\bar{r}^{2}\right)-\frac{\epsilon_{0} \epsilon_{r} \psi_{0} E_{S}}{\eta}\left(1-\frac{\bar{\psi}}{\bar{\psi}_{0}}\right) \text {. }
$$

Therefore, we may write

$$
\begin{aligned}
u_{\mathrm{avg}} & =\frac{\int_{0}^{R}\left(u_{P}+u_{E}\right) 2 \pi r d r}{\int_{0}^{R} 2 \pi r d r} \\
& =\frac{\int_{0}^{R} u_{P} 2 \pi r d r+\int_{0}^{R} u_{E} 2 \pi r d r}{\int_{0}^{R} 2 \pi r d r}=u_{\mathrm{avg}, P}+u_{\mathrm{avg}, E} \\
& =-\frac{1}{8 \eta} \frac{d p}{d x} R^{2}-\frac{\epsilon_{0} \epsilon_{r} \psi_{0} E_{S}}{\eta}\left[1-2 \bar{\lambda} \frac{I_{1}(1 / \bar{\lambda})}{I_{0}(1 / \bar{\lambda})}\right]
\end{aligned}
$$

Using Eqs. (20) and (6) in Eq. (19), we shall get

$$
u=2 u_{\mathrm{avg}, P}\left(1-\bar{r}^{2}\right)+\frac{u_{\mathrm{avg}, E}}{1-2 \bar{\lambda} \frac{I_{1}(1 / \bar{\lambda})}{I_{0}(1 / \bar{\lambda})}}\left[1-\frac{I_{0}(\bar{r} / \bar{\lambda})}{I_{0}(1 / \bar{\lambda})}\right] .
$$

We can relate $u_{\mathrm{avg}, E}$ to $u_{\mathrm{avg}, P}$ as [using Eq. (12) and the condition $-d p / d x=(2 \gamma / R) / \ell]$

$$
u_{\mathrm{avg}, E}=-8 \bar{\psi}_{0}^{2} N_{\lambda} u_{\mathrm{avg}, P},
$$

where

$$
N_{\lambda}=\left[1-2 \bar{\lambda} \frac{I_{1}(1 / \bar{\lambda})}{I_{0}(1 / \bar{\lambda})}\right] \bar{\lambda}^{2} \frac{\frac{I_{2}(1 / \bar{\lambda}) I_{1}(1 / \bar{\lambda})}{\left[I_{0}(1 / \bar{\lambda})\right]^{2}}}{\frac{S}{2}+\bar{\psi}_{0}^{2} M_{B}} .
$$

Hence, we can now write

$$
u_{\mathrm{avg}}=u_{\mathrm{avg}, P}+u_{\mathrm{avg}, E}=u_{\mathrm{avg}, P}\left[1-8 \bar{\psi}_{0}^{2} N_{\lambda}\right] .
$$

Therefore, the actual velocity profile can be expressed as

$$
\begin{aligned}
u= & 2 \frac{u_{\mathrm{avg}}}{\left[1-8 \bar{\psi}_{0}^{2} N_{\lambda}\right]}\left(1-\bar{r}^{2}\right)-\frac{u_{\mathrm{avg}}\left[8 \bar{\psi}_{0}^{2} N_{\lambda}\right]}{\left[1-8 \bar{\psi}_{0}^{2} N_{\lambda}\right]} \\
& \times \frac{1}{\left[1-2 \bar{\lambda} \frac{I_{1}(1 / \bar{\lambda})}{I_{0}(1 / \bar{\lambda})}\right]}\left[1-\frac{I_{0}(\bar{r} / \bar{\lambda})}{I_{0}(1 / \bar{\lambda})}\right] .
\end{aligned}
$$


From the above equation, we can write

$$
\begin{aligned}
\left(\frac{d u}{d r}\right)_{r=R}= & -\frac{4 u_{\mathrm{avg}}}{R\left[1-8 \bar{\psi}_{0}^{2} N_{\lambda}\right]}+\frac{1}{\bar{\lambda}} \frac{u_{\mathrm{avg}}\left[8 \bar{\psi}_{0}^{2} N_{\lambda}\right]}{R\left[1-8 \bar{\psi}_{0}^{2} N_{\lambda}\right]} \\
& \times \frac{1}{\left[1-2 \bar{\lambda} \frac{I_{1}(1 / \bar{\lambda})}{I_{0}(1 / \bar{\lambda})}\right]}\left[\frac{I_{1}(1 / \bar{\lambda})}{I_{0}(1 / \bar{\lambda})}\right] .
\end{aligned}
$$

Therefore, using Eq. (26) in Eq. (15) and $u_{\text {avg }}=d \ell / d t$ we get the drag force as

$$
F_{D}=-K_{2}^{\prime} \pi \eta \ell \frac{d \ell}{d t}
$$

where

$$
\begin{aligned}
K_{2}^{\prime}= & \frac{8}{1-8 \bar{\psi}_{0}^{2} N_{\lambda}}-\frac{1}{\bar{\lambda}} \frac{\left[16 \bar{\psi}_{0}^{2} N_{\lambda}\right]}{\left[1-8 \bar{\psi}_{0}^{2} N_{\lambda}\right]} \\
& \times \frac{1}{\left[1-2 \bar{\lambda} \frac{I_{1}(1 / \bar{\lambda})}{I_{0}(1 / \bar{\lambda})}\right]}\left[\frac{I_{1}(1 / \bar{\lambda})}{I_{0}(1 / \bar{\lambda})}\right] .
\end{aligned}
$$

The closed-form expression for $K_{2}^{\prime}$ provided in Eq. (28) is valid for small $\psi_{0}$. Please note that for generalized $\psi_{0}$, we shall get

$$
K_{2}^{\prime}=\frac{1-2 \bar{E}_{S}\left(\frac{d \bar{\psi}}{d \bar{r}}\right)_{\bar{r}=1}}{\frac{1}{8}-\bar{E}_{S} \bar{\psi}_{0}+\bar{E}_{S} \int_{0}^{1} \bar{\psi} \bar{r} d \bar{r}}
$$

where

$$
\bar{E}_{S}=\frac{1}{2} \frac{\int_{0}^{1} \sinh (\bar{\psi}) \bar{r}\left(1-\bar{r}^{2}\right) d \bar{r}}{S \int_{0}^{1} \cosh (\bar{\psi}) \bar{r} d \bar{r}+2 \bar{\psi}_{0} \int_{0}^{1}\left(1-\frac{\bar{\psi}}{\bar{\psi}_{0}}\right) \sinh (\bar{\psi}) \bar{r} d \bar{r}}
$$

is the expression for the dimensionless streaming potential used for numerical calculation (valid for any general $\psi_{0}$ ).

Length-versus-time relationship. Above, we have obtained all the components of the force balance equation [see Eqs. (1) and (2)] governing the capillary filling. Equation (2) can now be expressed in dimensionless form as

$$
\frac{d}{d \bar{t}}\left(\bar{\ell} \frac{d \bar{\ell}}{d \bar{t}}\right)=A-C \bar{\ell} \frac{d \bar{\ell}}{d \bar{t}}+A^{\prime}
$$

where $\bar{t}=t / \tau_{c}\left(\tau_{c}=\sqrt{\rho R^{3} / \gamma}\right.$ is the capillary time scale), $A=2 K_{1} \cos \theta, C=K_{2}^{\prime} \mathrm{Oh}(\mathrm{Oh}=\eta / \sqrt{\rho R \gamma}$ is the Ohnesorge number), and [using Eqs. (7) and (12)]

$$
A^{\prime}=\frac{q_{p} E_{S}}{\pi \gamma R}=-4 \frac{\bar{\psi}_{0}^{2} \bar{\lambda}}{S / 2+\bar{\psi}_{0}^{2} M_{B}} \frac{I_{1}(1 / \bar{\lambda}) I_{2}(1 / \bar{\lambda})}{I_{0}^{2}(1 / \bar{\lambda})}
$$

(please note $q_{0} E_{0} / \pi \gamma R=4$ ). For larger $\psi_{0}$, we obtain $A^{\prime}$ numerically.

Solving Eq. (29) analytically under the condition that the solution passes through zero and $\bar{\ell}_{\bar{t}=0}=0$, we can obtain the length-versus-time relationship as

$$
\bar{\ell}^{2}=\frac{2\left(A+A^{\prime}\right) \bar{t}}{C}-\frac{2\left(A+A^{\prime}\right)}{C^{2}}[1-\exp (-C \bar{t})] .
$$

Please note that for larger $\psi_{0}, A^{\prime}$ and $K_{2}^{\prime}$ (and hence $C$ ) are obtained numerically (as discussed above), but the analytical relationship expressed in Eq. (31) remains perfectly valid.

\section{RESULTS AND DISCUSSIONS}

The effect of charge on the capillary, which induces a streaming potential in the presence of the capillary transport, is manifested in two different ways. First, it imparts an electrical force on the liquid column, with $A^{\prime}$ being the dimensionless representation of this force. Second, it induces a back electroosmotic transport which changes the drag force, with the new drag force being quantified by the parameter $K_{2}^{\prime}$. These two factors combine to alter the overall $\ell$-vs- $t$ signature of the capillary filling process. In classical context, the streaming potential has mostly been described to induce this electroosmotic transport that eventually opposes the pressure-driven transport and in the process reduces the volume flow rate. However, this additional contribution of the electrical force arises from treating the filling capillary column as a lumped mass, as discussed earlier.

\section{A. Variation of the electrical force on the liquid column}

Electrical force exerted on the liquid column is a combination of two components: the streaming potential $E_{S}$ and the net EDL charge $q_{p}$. Variations of both of them, in dimensionless forms, as functions of the dimensionless EDL thickness $\bar{\lambda}$ (please note that in an experiment one can increase $\bar{\lambda}$ either by lowering the ion concentration or by reducing the capillary radius), are depicted in Fig. 2(a). The dimensionless streaming potential $\left(\bar{E}_{S}\right)$ first increases (in magnitude) with $\bar{\lambda}$, ultimately attaining saturation at a larger overlapped value of $\bar{\lambda}(\bar{\lambda}=1$ signifies the start of the EDL overlap; Ref. [72] also reports such a behavior). Such a trend can be explained from the functional dependence of $\bar{E}_{S}$ on the EDL potential $\bar{\psi}$ and hence $\bar{\lambda}$ [see Eq. (12)]. For thicker EDLs, there is a finite value of the EDL potential even at larger distances from the capillary wall. This enhances the streaming current, which is proportional to $\int_{0}^{R} r\left(1-r^{2} / a^{2}\right) \psi d r$. In case $\bar{\lambda}$ becomes substantially large (i.e., when EDLs overlap), $\bar{\psi}$ attains a uniform value (equal to the dimensionless wall potential $\bar{\psi}_{0}$, we represent this condition mathematically as $\bar{\psi} \rightarrow \bar{\psi}_{0}$ ) across the entire capillary cross section, and therefore ceases to vary with $\bar{\lambda}$. This causes the streaming potential to saturate at a given value at large overlapped $\bar{\lambda}$ values. The second issue concerning $\bar{E}_{S}$ is its dependence on the dimensionless ionic Peclet number $S$ (see Ref. [91] for an estimate of $S$ ). $\bar{E}_{S}$ decreases monotonically with $S$, and such a dependence can be explained following the analysis presented in several other studies [63,65]. The magnitude of the net dimensionless charge contained in the liquid column $\left|\bar{q}_{p}\right|$ [see Eq. (7)] decreases monotonically with $\bar{\lambda}$ [see the inset of Fig. 2(a) and the discussions following Eq. (7)]. In Fig. 2(a) we show the variation of $E_{S}$ and $q_{p}$ for both small $\psi_{0}$ (for which we get closed-form analytical results) and larger $\psi_{0}$ (for which we need numerical results). For larger $\psi_{0}, E_{S}$ gets reduced (see Ref. [63] for a detailed explanation), but $\left|q_{p}\right|$ is substantially enhanced because of a larger counterion concentration required to screen the larger wall charge.

$A^{\prime}$ is the dimensionless quantification of the net electrical force. $A^{\prime}$ is a product of $E_{S}$ and $q_{p}$, with the product made dimensionless with the surface tension force $\gamma \pi R . A^{\prime}$ first increases (in magnitude) with $\bar{\lambda}$ (for small $\bar{\lambda}$ values) manifesting a more dominating influence of the streaming 


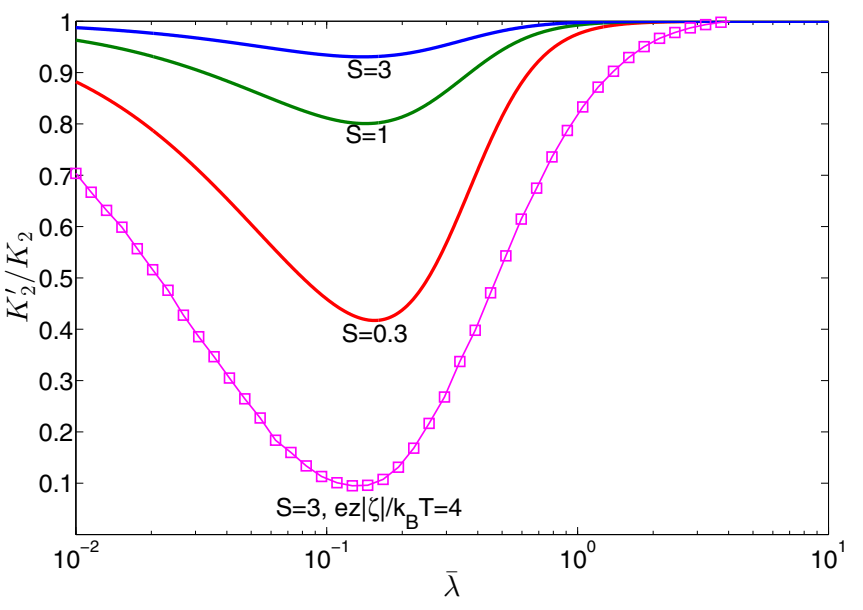

FIG. 3. (Color online) Variation of the dimensionless ratio $K_{2}^{\prime} / K_{2}$ with the dimensionless EDL thickness $\bar{\lambda}$ for different values of the dimensionless ionic Peclet number $S$. The ratio $K_{2}^{\prime} / K_{2}$ is equal to the ratio $\eta / \eta_{\text {app }}$, where $\eta_{\text {app }}$ is the apparent viscosity characterizing the electroviscous effect. Except for the case where $\left|\bar{\psi}_{0}\right|=e z\left|\psi_{0}\right| / k_{B} T$ is specified, we always take $\left|\bar{\psi}_{0}\right|=1$. For the case where $\left|\bar{\psi}_{0}\right|=4$, we obtain the results numerically.

potential. However, beyond a critical $\bar{\lambda}$, the effect of $\left|\overline{q_{p}}\right|$ becomes more dominant, since for larger $\bar{\lambda}, \bar{E}_{S}$ ceases to increase with $\bar{\lambda}$. This ensures a decrease in $A^{\prime}$ with $\bar{\lambda}$, ultimately becoming negligible at large overlapped $\bar{\lambda}$ values. Another important observation is that the sign of the dimensionless electrical force is always negative (independent of the sign of the wall potential), indicating that the net electrical force always opposes the capillary filling process. This occurs since when $\bar{\psi}_{0}>0, \bar{E}_{S}>0$ and $\bar{q}_{p}<0$ (since the counterions are negative), whereas when $\bar{\psi}_{0}<0, \bar{E}_{S}<0$ and $\bar{q}_{p}>0$ (since the counterions are positive).

\section{B. Variation of the drag force}

Figure 3 shows the variation of the parameter $K_{2}^{\prime} / K_{2}$, signifying the alteration in the drag force on account of considering a combined pressure-driven and electro-osmotic transport. This drag force is the wall-induced drag, which is solely a function of the velocity profile. Hence a larger net velocity will imply a larger value of this drag force. This ratio $K_{2}^{\prime} / K_{2}$ is always less than unity. This signifies that the net drag force of the combined transport is always lower than that of a pure pressure-driven transport. This occurs as the streaming-potential-induced electro-osmotic transport opposes the pressure-driven capillary transport. For smaller $\bar{\lambda}$, the ratio $K_{2}^{\prime} / K_{2}$ decreases with $\bar{\lambda}$, manifesting a larger influence of the retarding electro-osmotic transport. However, beyond a critical $\bar{\lambda}$ value, $K_{2}^{\prime} / K_{2}$ increases with $\bar{\lambda}$, finally becoming 1. This signifies that for large extents of EDL overlap, there is no effect of the retarding electro-osmotic transport. This electro-osmotic transport, which causes the lowering of the drag, depends on the streaming potential $\bar{E}_{S}$ and the electro-osmotic flow profile that varies as $1-\bar{\psi} / \bar{\psi}_{0}$. $\bar{E}_{S}$ increases with $\bar{\lambda}$, and this increase, particularly for small $\bar{\lambda}$ [see Fig. 2(a)], is responsible for an equivalent decrease in the drag coefficient for such $\bar{\lambda}$ values. However, at larger $\bar{\lambda}, \bar{E}_{S}$ ceases to vary with $\bar{\lambda}$ [see Fig. 2(a)], whereas the corresponding electro-osmotic velocity $\left[\sim\left(1-\bar{\psi} / \bar{\psi}_{0}\right)\right]$ would tend to zero (since $\bar{\psi} \rightarrow \bar{\psi}_{0}$ ). Therefore, the electro-osmotic flow gets weakened leading to an increase in the ratio $K_{2}^{\prime} / K_{2}$ to a value close to unity for larger $\bar{\lambda}$ values. Key to note here is that the critical $\bar{\lambda}$ value beyond which $K_{2}^{\prime} / K_{2}$ starts to increase is different from the $\bar{\lambda}$ value beyond which $\left|A^{\prime}\right|$ starts to decrease. This can be justified by noting that the magnitude of dimensionless net charge $\left|\bar{q}_{p}\right|$ and the electro-osmotic flow profile $1-\bar{\psi} / \bar{\psi}_{0}$ demonstrate different functional dependences on $\bar{\lambda}$, although both decrease monotonically with $\bar{\lambda}$. Please note that this ratio $K_{2}^{\prime} / K_{2}$ is equal to the ratio $\eta / \eta_{\text {app }}$, where $\eta_{\text {app }}$ is the apparent viscosity characterizing the electroviscous effect. Here too we provide results for both small and large $\bar{\psi}_{0}$. Electro-osmotic flow velocity $\sim \bar{\psi}_{0}$, and hence larger $\bar{\psi}_{0}$ enhances the streaming-potential-induced retarding electro-osmotic transport, causing a weaker net flow and hence a weaker drag (quantified by a weaker $K_{2}^{\prime} / K_{2}$ ratio).

\section{Capillary filling dynamics}

In this section, we shall discuss the central results of the paper, namely, the role of charge on the capillary walls in affecting the filling dynamics. Figure 4 clearly shows that for charged capillaries, the filling speed is reduced, as evidenced by a smaller dimensionless filling length $\bar{\ell}$ for a given

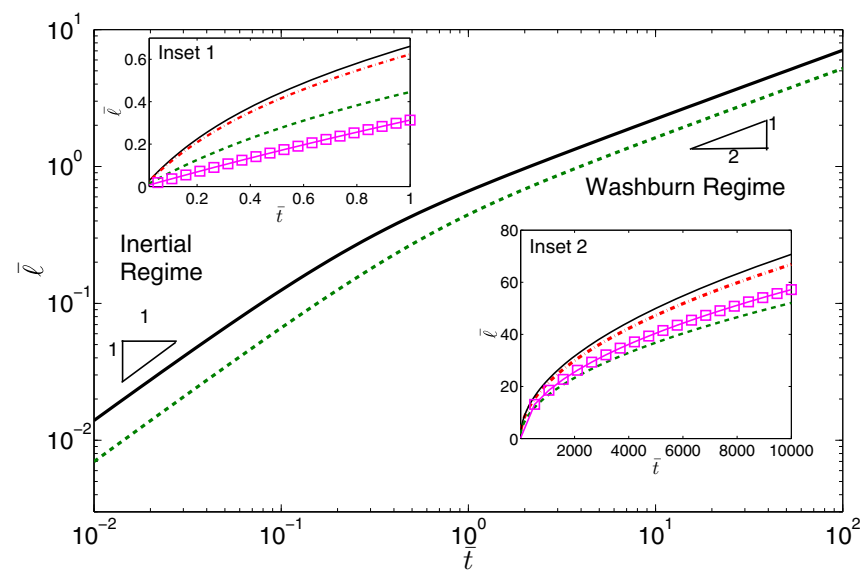

FIG. 4. (Color online) Variation of $\bar{\ell}$-vs- $\bar{t}$ for uncharged and charged capillaries. In the plot, we indicate the initial inertial regime $[23,30]$ (where $\bar{\ell} \sim \bar{t}$ ) and the Washburn regime [2,3,15-23,30,37,59] (where $\bar{\ell} \sim \sqrt{\bar{t}}$ ). $\ell$-vs- $t$ behavior for these two regimes is separately magnified in insets 1 and 2 of the plot. The results for charged capillaries are provided for different values of $S$. We choose dimensionless EDL thickness $\bar{\lambda}$ values, corresponding to these $S$ values, such that the magnitude of the electric force on the liquid column [see Fig. 2(b)] is maximum. In the main plot and the plots in the inset, we use a bold line for the uncharged capillary and a dashed line for the charged capillary $(S=0.3$ and $\bar{\lambda}=0.2178$ ). For the plots in the inset, dashed-dotted lines represent the case for the charged capillary $(S=3$ and $\bar{\lambda}=0.2911)$, whereas the plot with square markers represents the case corresponding to larger $\left|\bar{\psi}_{0}\right|$ ( $S=3$ and $\bar{\lambda}=0.1265$ ). All the results are shown for $\mathrm{Oh}=1$, $A=2 K_{1} \cos \theta=2$, and $\left|\bar{\psi}_{0}\right|=1$ (except for the case of larger $\left|\bar{\psi}_{0}\right|$, where we take $\left.\left|\bar{\psi}_{0}\right|=4\right)$. 


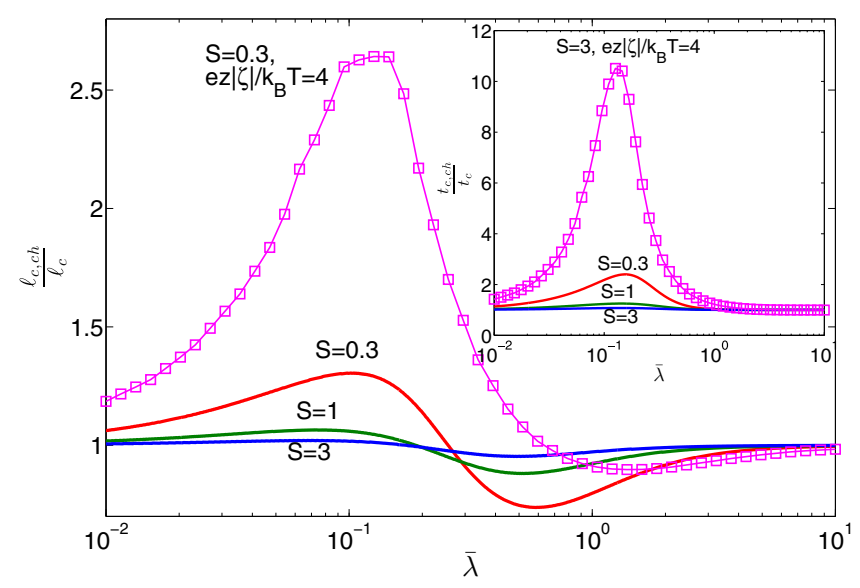

FIG. 5. (Color online) Variation of the ratio $\bar{\ell}_{c, \mathrm{ch}} / \bar{\ell}_{c}=\ell_{c, \mathrm{ch}} / \ell_{c}$ with the dimensionless EDL thickness $\bar{\lambda}$ for different values of the dimensionless ionic Peclet number $S$. The plotted ratio is independent of Oh, although $\bar{\ell}_{c}$ and $\bar{\ell}_{c, \text { ch }}$ depend on it. For example, we have $\bar{\ell}_{c}=0.2964$ (for $\mathrm{Oh}=1$ ) and $\bar{\ell}_{c}=2.964$ (for $\mathrm{Oh}=0.1$ ), so that the linear regime can be defined for $\bar{\ell}_{c} \times \mathrm{Oh} \ll 1$ [30] (and the transition from a linear to Washburn regime would occur for $\bar{\ell}_{c} \times \mathrm{Oh} \sim 1[30]$ ). In the inset of the figure, we plot the ratio $\bar{t}_{c, \mathrm{ch}} / \bar{t}_{c}=t_{c, \mathrm{ch}} / t_{c}$ with $\bar{\lambda}$ for different values of $S$. Here both $\bar{t}_{c, \text { ch }}$ and $\bar{t}_{c}$ are independent of Oh, and we get $\bar{t}_{c}=t / \tau_{c}=0.2883$, i.e., the linear regime is witnessed for $t \ll \tau_{c}$ and the transition from the linear to Washburn regime occurs for $t \sim \tau_{c}$ [30]. For these plots, except for the case where $\left|\bar{\psi}_{0}\right|=e z\left|\psi_{0}\right| / k_{B} T$ is specified, we always take $\left|\bar{\psi}_{0}\right|=1$. For the case where $\left|\bar{\psi}_{0}\right|=4$, we obtain the results numerically.

dimensionless time $\bar{t}$. This becomes possible since the effect of the retarding electrical force on the filling liquid column substantially overweighs the consequence of reduced drag due to back electro-osmotic transport. However, the presence of the charge on the capillary does not affect the appearance of the linear and the Washburn regimes that characterize the classical horizontal capillary filling process. In the two insets of Fig. 4, we have separately magnified the $\bar{\ell}$-vs- $\bar{t}$ behavior for the linear and the Washburn regimes for the charged and the uncharged capillaries. The results in Fig. 4 as well as those in the two insets are shown for different $S$ values. Also for these figures, the dimensionless EDL thickness value is so chosen that, corresponding to the chosen $S$, the electrical force on the liquid column [see Fig. 2(b)] is maximum. Results are provided for both small and large $\bar{\psi}_{0}$. An increase in $S$ lowers the electrical force, and this is manifested through a smaller difference in the $\bar{\ell}$-vs- $\bar{t}$ variation between the charged and the uncharged capillaries. Larger $\bar{\psi}_{0}$ leads to a larger magnitude of the retarding electrical force [see Fig. 2(b)], causing a larger value of this difference (for identical $S$ ).

Figure 5 and its inset demonstrate the consequence of charge on the capillary in affecting the critical capillary filling length $\left(\ell_{c}\right)$ and the critical filling time $\left(t_{c}\right)$ corresponding to which the $\ell$-vs- $t$ variation transits from the linear to the Washburn regime. Here we denote $\ell_{c, \text { ch }}$ and $t_{c, \text { ch }}$ as values corresponding to the case of the charged capillary. From the analytical $\ell$-vs- $t$ relationship [see Eq. (31)], we can demarcate the linear regime as the one where $\exp (-C \bar{t}) \ll 1$, i.e.,
$|C \bar{t}| \ll 1$, since for such a case we can expand Eq. (31) as

$$
\begin{aligned}
\bar{\ell}^{2} & \approx \frac{2\left(A+A^{\prime}\right) \bar{t}}{C}-\frac{2\left(A+A^{\prime}\right)}{C^{2}}\left[1-\left(1-C \bar{t}+\frac{1}{2} C^{2} \bar{t}^{2}\right)\right] \\
& \Rightarrow \bar{\ell}^{2} \approx\left(A+A^{\prime}\right) \bar{t}^{2} \Rightarrow \bar{\ell} \approx \sqrt{A+A^{\prime}} \bar{t}
\end{aligned}
$$

For the present case, we consider that such a purely linear regime ceases to exist for $\exp (-C \bar{t})<0.1$. Therefore, $t_{c}$ or $t_{c, \text { ch }}$ is considered as the time such that $\exp \left(-C \bar{t}_{c}\right)<0.1$ and $\exp \left(-C \bar{t}_{c, \mathrm{ch}}\right)<0.1$. The corresponding values of the filling length are considered as $\bar{\ell}_{c}$ and $\bar{\ell}_{c, \text { ch. }}$. Using this description, we can clearly justify $\bar{t}_{c \text {,ch }} / \bar{t}_{c} \sim K_{2} / K_{2}^{\prime}$ (since $C=K_{2} \mathrm{Oh}$ ), and this can be witnessed in the figure in the inset of Fig. 5. This figure also shows that the ratio $\bar{t}_{c, \mathrm{ch}} / \bar{t}_{c}$ is always greater than unity, indicating a delay in the linear-to-Washburn regime transition for the charged capillary. This ratio, at a given $\bar{\lambda}$, is always larger for a smaller $S$. Also for larger $\bar{\psi}_{0}$, this ratio is substantially augmented, indicating a much weaker filling dynamics caused by the presence of a much larger retarding electrical force [see Fig. 2(b)]. Further, $\bar{t}_{c, \text { ch }} / \bar{t}_{c}$ first increases with $\bar{\lambda}$, attains a maximum, and then decreases with the EDL thickness. All these variations exactly follow the inverse behavior of the ratio $K_{2}^{\prime} / K_{2}$ with $S$ and $\bar{\lambda}$ (see Fig. 3). A variation of the corresponding filling length, quantified by the ratio $\bar{\ell}_{c, \mathrm{ch}} / \bar{\ell}_{c}$ (see Fig. 5), on the other hand, cannot be inferred so trivially. For a given time, $\bar{\ell}$ is always smaller for a charged capillary (see Fig. 4). However, when we are looking at two disparately spaced times, i.e., we are studying the filling length for a time that is much larger for the case of charged capillary, it becomes possible that the corresponding filling length for the charged capillary is more than that for the uncharged case. This is precisely the case for smaller $\bar{\lambda}$. Here the ratio $\bar{\ell}_{c, \mathrm{ch}} / \bar{\ell}_{c}$ is more than unity, indicating that for a charged capillary the linear-to-Washburn regime transition occurs at a larger filling length. For larger $\bar{\psi}_{0}$, this ratio is even higher, indicating a significantly retarded transport caused by the larger retarding electrical force [see Fig. 2(b)]. This ratio $\left(\bar{\ell}_{c, \mathrm{ch}} / \bar{\ell}_{c}\right)$ also increases with $\bar{\lambda}$ for small $\bar{\lambda}$, reaches a maximum, and then starts to decrease with $\bar{\lambda}$ for larger $\bar{\lambda}$. More importantly, for larger values of $\bar{\lambda}$, this ratio becomes less than unity. This indicates that, for a charged capillary, the linear-to-Washburn regime transition is occurring at a filling length that is smaller than that for an uncharged capillary. Subsequently, this ratio attains a minimum for a substantially large EDL thickness. This represents the situation where there is an imperceptible difference between $\bar{t}_{c \text {,ch }}$ and $\bar{t}_{c}$, and accordingly the effect of reduced driving force dominates, resulting in smaller values of the transition filling lengths for the charged capillary. Finally, for very large extents of EDL overlap, the ratio becomes unity, since for such an EDL thickness value both the net electrical force and the reduced drag are of little or no significance.

\section{Neglect of the charge accumulation at the air-liquid interface}

One of the main assumptions in our calculation is the neglect of charge accumulation at the air-liquid interface. In order to understand the impact of such an assumption, it is important to first pinpoint why such an accumulation will 
occur. Axial charge separation is essential for the generation of a streaming potential. At steady state in a micronanochannel charge is accumulated at the ends of the channel, and the rate of accumulation of that charge (the streaming potential is proportional to this rate of accumulation) is obtained by calculating the advective transport of the charge density gradient. On the contrary, as has been identified by Phan et al. [61], for the capillary filling problem charge will accumulate at the traveling air-liquid meniscus. Phan et al. [61] attempted to model this situation by equating the sum of the streaming and conduction currents not to zero, but to the rate of charge accumulation at the interface. This is indeed a novel method to account for the charge accumulation at the air-liquid interface. However, the main limitation of this approach [61] is that it does not account for the fact that such an accumulation of charges at the interface will imply that the electrostatic potential and the ion number distributions become two dimensional. In a future study, we shall attempt to provide a complete numerical solution where such charge accumulation at the interface is accounted for in the presence of the following considerations: (a) twodimensional distribution of the electrostatic potential and the ion number, (b) two-dimensional velocity fields resulting from a two-dimensional electrostatic potential, and (c) varying the shape of the interface on account of the movement of the interface (i.e., using a dynamic contact angle). Such a detailed analysis is beyond the scope of the present study, which focuses on identifying the important physical effects involved in filling of a charged capillary. Of course, our calculations remain approximate, given the fact that our
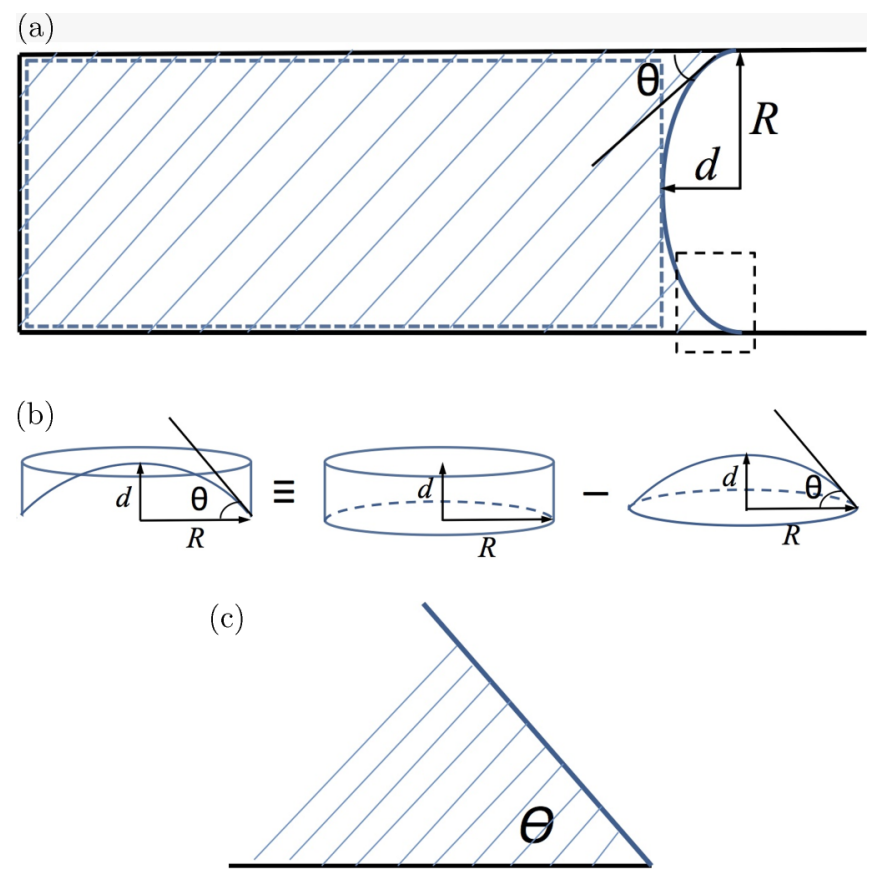

FIG. 6. (Color online) (a) Schematic of the capillary filling. The control volume denoted with the dotted line is the filling liquid column that we analyze. (b) The volume of the liquid at the interface $\left(V_{\text {int }}\right)$ that we neglect in our analysis. $V_{\text {int }}$ is equal to the volume of the cylinder minus the volume of the spherical cap. (c) Wedge at the three-phase contact line indicated in (a). computation of the streaming potential (owing to neglecting the charge accumulation at the meniscus) and the electrostatic, ion number density, and velocity distributions (because we consider them as one dimensional) remain approximate.

The second and more intuitive situation where charges can be present at the air-liquid interface is the case where the EDL is thick (and comparable to the capillary radius) and $0<\theta<\pi / 2$. In such a scenario, the wedge formed by the three-phase contact line [see Fig. 6(a)] will consist of two charged interfaces: a charged solid-liquid interface and a charged air-liquid interface. Also, such a consideration, such as the previous case, will necessitate a two-dimensional description of all the variables (electrostatic potential, ion number densities, and velocity field). In the present calculation, we neglect this wedge effect; as shown in the Appendix, such an approximation remains valid as long as $R / \ell$ and/or $\theta$ are small.

As shown in Ref. [79], the EDL in the vicinity of such a wedge-shaped TPCL would be nontrivially different from the one-dimensional EDL distribution, and would substantially alter the present calculations. More importantly, the electroosmotic flow field in such a geometry remains unknown, particularly given the large singularity associated with the stress for $r \rightarrow 0$ at the TPCL. Therefore, the first step to incorporate the wedge effects in the present calculations would be to pinpoint the electro-osmotic flow profile in the wedge. Such an endeavor is beyond the scope of the present calculations.

\section{CONCLUSIONS}

In this paper, we have shown that the filling of charged capillaries may be characterized by two distinct effects that are absent in uncharged capillaries. The first effect is the retarding electrical force on the filling liquid column and the second effect is the reduced drag force because of the induced back electro-osmotic transport. The former effect overwhelms the latter, so that the net consequence is a weaker filling rate and a slower transition from the linear to the Washburn regime; however, the corresponding transition filling length, depending on the EDL thickness, wall potential, and dimensionless ionic Peclet number, may be larger or smaller than that corresponding to an uncharged capillary.

The findings offered by the present study are especially significant on two accounts. First, it allows a substantially better understanding of the standard filling processes used in experiments, e.g., filling of glass or silicon microcapillaries. Such microcapillaries are often inherently charged or may acquire charges due to spontaneous de-ionization by the filling liquid. As a result, even without the application of an external electric field, the filling process may get substantially altered on account of the induced streaming potential and the consequent electroresistive forces and back-electro-osmosis-driven reduced drag forces. Second, the present analysis provides a mechanism of controlling the lifespan of different regimes associated with the classical capillary filling process. Such control may be desirable in different applications demanding a specified $\ell$-vs- $t$ behavior in the capillary filling process. These two factors establish the relevance of the present study in better 
understanding the design and operation of microfluidics-based laboratory-on-a-chip devices.

\section{ACKNOWLEDGMENT}

A part of this work was done when S.D. was at the University of Alberta, Canada.

\section{APPENDIX: VOLUME OF CHARGE CONTAINING INTERFACE NEGLECTED IN THE PRESENT ANALYSIS}

We consider a wetting liquid, i.e., $\theta<\pi / 2$ [see Fig. 6(a)]. From Fig. 6(b), we can see that the volume of the charge containing interface $\left(V_{\text {int }}\right)$ is equal to the volume of cylinder $V_{\text {cyl }}$ (with height $d$ and radius $R$ ) minus the volume of the spherical cap $\left(V_{s}\right)$ with height $d$, contact angle $\theta$, and base radius $R$. From Fig. 6(b), we get

$$
d=R_{0}(1-\cos \theta)=R \frac{1-\cos \theta}{\sin \theta}=R \tan \frac{\theta}{2} .
$$

Consequently,

$$
V_{\mathrm{cyl}}=\pi R^{2} d=\pi R^{3} \tan \frac{\theta}{2}
$$

and

$$
V_{s}=\frac{\pi d}{6}\left(3 R^{2}+d^{2}\right)=\frac{\pi R^{3}}{6} \tan \frac{\theta}{2}\left(3+\tan ^{2} \frac{\theta}{2}\right) .
$$

Therefore,

$$
V_{\mathrm{int}}=V_{\mathrm{cyl}}-V_{s}=\pi R^{3} \tan \frac{\theta}{2}\left(\frac{1}{2}-\frac{1}{6} \tan ^{2} \frac{\theta}{2}\right) .
$$

This is the charge contained in the liquid volume (at the air-liquid interface) resulting from consideration of the wedge geometry at the three-phase contact line, especially relevant for thick EDLs and $0<\theta<\pi / 2$. This charge, therefore, is the charge of the EDL in a wedgelike volume [79,92], and is different from the charge that accumulates at the meniscus on account of the axial separation of charges (responsible for triggering the streaming potential). We have neglected this charge in the present analysis. Comparing this charge containing volume with the volume of the cylindrical filling liquid column (of volume $V_{\text {fill }}=\pi R^{2} \ell$ ), we can easily see that

$$
\frac{V_{\text {int }}}{V_{\text {fill }}}=\frac{R}{\ell} \tan \frac{\theta}{2}\left(\frac{1}{2}-\frac{1}{6} \tan ^{2} \frac{\theta}{2}\right)
$$

Thus, if $R \ll \ell$ or $\theta \rightarrow 0$, our approximation of neglecting the effect of this charge at the meniscus remains valid.
[1] R. Lucas, Kolloid Z. 23, 15 (1918).

[2] E. W. Washburn, Phys. Rev. 17, 273 (1921).

[3] C. H. Bosanquet, Philos. Mag. 45, 525 (1923).

[4] A. L. Betz and G. W. Goldstein, Annu. Rev. Phys. 48, 241 (1986).

[5] C. Heltianu, L. Dobrila, F. Antohe, and M. Simionescu, Microvasc. Res. 37, 188 (1989).

[6] R. Skalak, N. Ozkaya, and T. C. Skalak, Annu. Rev. Fluid Mech. 21, 167 (1989).

[7] K. Kroll, T. R. Bukowski, L. M. Schwartz, D. Knoepfler, and J. B. Bassingthwaighte, Am. J. Physiol. Heart Circ. Physiol. 262, H420 (1992).

[8] S. Chakraborty, Lab Chip 5, 421 (2005).

[9] S. Chakraborty, Anal. Chim. Acta 605, 175 (2007).

[10] E. Kim, Y. Xia, and G. M. Whitesides, J. Am. Chem. Soc. 118, 5722 (1996).

[11] M. Zimmerman, H. Schmid, P. Hunziker, and E. Delamarche, Lab Chip 7, 119 (2007).

[12] A. W. Martinez, S. T. Philips, and G. M. Whitesides, Proc. Natl. Acad. Sci. USA 105, 19606 (2008).

[13] L. Hu, H. Wu, F. L. Fantia, Y. Yang, and Y. Cui, ACS Nano 4, 5843 (2010).

[14] Y. Yagi, S. Yamamoto, K. Kakehi, T. Hayakawa, Y. Ohyama, and S. Suzuki, Electrophoresis 32, 2979 (2011).

[15] B. V. Zhmud, F. Tiberg, and K. Hallstensson, J. Colloid Interface Sci. 228, 263 (2000).

[16] A. Han, G. Mondin, N. G. Hegelbach, N. F. de Rooij, and U. Staufer, J. Colloid Interface Sci. 293, 151 (2006).

[17] J. Haneveld, N. R. Tas, N. Brunets, H. V. Jansen, and M. Elwenspoek, J. Appl. Phys. 104, 014309 (2008).

[18] J. M. Oh, T. Faez, S. de Beer, and F. Mugele, Microfluid. Nanofluid. 9, 123 (2010).
[19] P. R. Waghmare and S. K. Mitra, Microfluid. Nanofluid. 12, 53 (2012).

[20] P. R. Waghmare and S. K. Mitra, Anal. Chim. Acta 663, 117 (2010).

[21] A. Saha and S. K. Mitra, J. Colloid Interface Sci. 339, 461 (2009).

[22] A. Saha, S. K. Mitra, M. Tweedie, S. Roy, and J. McLaughlin, Microfluid. Nanofluid. 7, 451 (2009).

[23] D. Quere, Eur. Phys. Lett. 39, 533 (1997).

[24] A. Kutana and K. P. Giapis, Nano Lett. 6, 656 (2006).

[25] D. I. Dimitrov, A. Milchev, and K. Binder, Phys. Rev. Lett. 99, 054501 (2007).

[26] N. Ichikawa and Y. Satoda, J. Colloid Interface Sci. 162, 350 (1994).

[27] M. Dreyer, A. Delgado, and H. Rath, J. Colloid Interface Sci. 163, 158 (1994).

[28] L. Joly, J. Chem. Phys. 135, 214705 (2011).

[29] S. Supple and N. Quirke, Phys. Rev. Lett. 90, 214501 (2003).

[30] S. Das, P. R. Waghmare, and S. K. Mitra, Phys. Rev. E 86, 067301 (2012).

[31] B. Lavi, A. Marmur, and J. Bachmann, Langmuir 24, 1918 (2008).

[32] N. Fries and M. Dreyer, J. Colloid Interface Sci. 338, 514 (2009).

[33] N. Fries and M. Dreyer, J. Colloid Interface Sci. 327, 125 (2008).

[34] D. Quere and E. Raphaël, Langmuir 15, 3679 (1999).

[35] E. Lorenceau, D. Quere, J.-Y. Ollitrault, and C. Clanet, Phys. Fluids 14, 1985 (2002).

[36] R. Masoodi, E. Languri, and A. Ostadhossein, J. Colloid Interface Sci. 389, 268 (2013).

[37] S. Das and S. K. Mitra, Phys. Rev. E 87, 063005 (2013).

[38] J. Szekely, A. W. Neumann, and Y. K. Chuang, J. Colloid Interface Sci. 35, 273 (1971). 
[39] M. Stange, M. E. Dreyer, and H. J. Rath, Phys. Fluids 15, 2587 (2003).

[40] J. van Brakel and P. M. Heertjes, Nature (London) 254, 585 (1975).

[41] T. Delker, D. B. Pengra, and P. Z. Wong, Phys. Rev. Lett. 76, 2902 (1996).

[42] M. Lago and M. Araujo, Physica A 289, 1 (2001).

[43] A. Hamraoui and T. Nylander, J. Colloid Interface Sci. 250, 415 (2002).

[44] B. J. Mullins and R. D. Braddock, Int. J. Heat Mass Transfer 55, 6222 (2012).

[45] K. S. Sorbie, Y. Z. Wu, and S. R. McDougall, J. Colloid Interface Sci. 174, 289 (1995).

[46] F. Maggi, Colloids Surf., A 415, 119 (2012).

[47] S. Chibbaro, L. Biferale, F. Diotallevi, and S. Succi, Eur. Phys. J. Spec. Top. 171, 223 (2009).

[48] J. Bico and D. Quere, J. Colloid Interface Sci. 247, 162 (2002).

[49] R. Holtzman, M. L. Szulczewski, and R. Juanes, Phys. Rev. Lett. 108, 264504 (2012).

[50] D. Erickson, D. Li, and C. B. Park, J. Colloid Interface Sci. 250, 422 (2002).

[51] Z. Wang, C. C. Chang, S. J. Hong, Y. J. Sheng, and H. K. Tsao, Langmuir 28, 16917 (2012).

[52] S. Chakraborty and R. Mittal, J. Appl. Phys. 101, 104901 (2007).

[53] D. Chakraborty and S. Chakraborty, Langmuir 24, 9449 (2008).

[54] F. Mugele and J.-C. Baret, J. Phys.: Condens. Matter 17, R705 (2005).

[55] W. C. Nelson and C.-J. Kim, J. Adhes. Sci. Technol. 26, 1747 (2012).

[56] S. Das, S. Chakraborty, and S. K. Mitra, Phys. Rev. E 85, 046311 (2012).

[57] S. Das, S. K. Mitra, and S. Chakraborty, Phys. Rev. E 86, 011603 (2012).

[58] S. Das, S. K. Mitra, and S. Chakraborty, Phys. Rev. E 86, 056317 (2012).

[59] N. R. Tas, J. Haneveld, H. V. Jansen, M. Elwenspoek, and A. van den Berg, Appl. Phys. Lett. 85, 3274 (2004).

[60] F. Persson, L. H. Thamdrup, M. B. L. Mikkelsen, S. E. Jaarlgard, P. Skafte-Pedersen, H. Bruus, and A. Kristensen, Nanotechnology 18, 245301 (2007).

[61] V.-N. Phan, C. Yang, and N.-T. Nguyen, Microfluid. Nanofluid. 7, 519 (2009).

[62] D. Burgreen and F. R. Nakache, J. Phys. Chem. 68, 1084 (1964); C. L. Rice and R. Whitehead, ibid. 69, 4017 (1965); S. Levine, J. R. Marriott, G. Neale, and N. Epstein, J. Colloid Interface Sci. 52, 136 (1975).
[63] S. Chakraborty and S. Das, Phys. Rev. E 77, 037303 (2008).

[64] S. Das and S. Chakraborty, Langmuir 25, 9863 (2009).

[65] S. Das and S. Chakraborty, Langmuir 26, 11589 (2010).

[66] L. Ren, D. Li, and W. Qu, J. Colloid Interface Sci. 233, 12 (2001).

[67] T. Das, S. Das, and S. Chakraborty, J. Chem. Phys. 130, 244904 (2009).

[68] S. Chanda, S. Sinha, and S. Das, Soft Matter 10, 7558 (2014).

[69] M. Wang, C.-C. Chang, and R.-J. Yang, J. Chem. Phys. 132, 024701 (2010).

[70] N. A. Mortensen and A. Kristensen, Appl. Phys. Lett. 92, 063110 (2008).

[71] A. Jain and S. Chakraborty, Anal. Chim. Acta 659, 1 (2010).

[72] S. Das, A. Guha, and S. K. Mitra, Anal. Chim. Acta 804, 159 (2013).

[73] R. J. Hunter, Zeta Potential in Colloid Science (Academic, London, 1981).

[74] K. Kang, I. Kang, and C. Lee, Langmuir 19, 5407 (2003).

[75] K. Kang, I. Kang, and C. Lee, Langmuir 19, 6881 (2003).

[76] K. Kang, I. Kang, and C. Lee, Langmuir 19, 9334 (2003).

[77] K. Kang and I. Kang, Langmuir 19, 9962 (2003).

[78] C. K. Hua, I. S. Kang, K. H. Kang, and H. A. Stone, Phys. Rev. E 81, 036314 (2010).

[79] A. Dörr and S. Hardt, Phys. Rev. E 86, 022601 (2012).

[80] B. D. Storey, L. R. Edwards, M. S. Kilic, and M. Z. Bazant, Phys. Rev. E 77, 036317 (2008).

[81] M. Z. Bazant, M. S. Kilic, B. D. Storey, and A. Ajdari, Adv. Colloid Interface Sci. 152, 48 (2009).

[82] S. Das and S. Chakraborty, Phys. Rev. E 84, 012501 (2011).

[83] S. Das, Phys. Rev. E 85, 012502 (2012).

[84] S. Chanda and S. Das, Phys. Rev. E 89, 012307 (2014).

[85] S. Das, S. Chakraborty, and S. K. Mitra, Phys. Rev. E 85, 051508 (2012).

[86] R. P. Misra, S. Das, and S. K. Mitra, J. Chem. Phys. 138, 114703 (2013).

[87] S. H. Behrens and D. G. Grier, J. Chem. Phys. 115, 6716 (2001).

[88] F. H. J. van der Heyden, D. Stein, and C. Dekker, Phys. Rev. Lett. 95, 116104 (2005).

[89] S. Das, K. Subramanian, and S. Chakraborty, Colloids Surf., B 58, 203 (2007).

[90] S. Das, Phys. Rev. E 84, 036303 (2011).

[91] Considering water as the solvent (so that $\epsilon_{r}=79.8$ ) and $\mathrm{NaCl}$ as added salt (so that $R_{\text {ion }} \approx 0.3 \mathrm{~nm}$ and $z=1$ ), we get (using $\left.f \sim 6 \pi \eta R_{\text {ion }}\right) S \approx 3$.

[92] S. Das and S. K. Mitra, Phys. Rev. E 88, 033021 (2013). 